\title{
A benchmark study of the Signed-particle Monte Carlo algorithm for the Wigner equation
}

\author{
Orazio Muscato ${ }^{1^{*}}$ \\ ${ }^{1}$ Dipartimento di Matematica e Informatica \\ Università degli Studi di Catania, Italy \\ *Email address for correspondence: muscato@dmi.unict.it \\ Communicated by Giovanni Russo \\ Received on 12 09, 2016. Accepted on 10 12, 2017.
}

\begin{abstract}
The Wigner equation represents a promising model for the simulation of electronic nanodevices, which allows the comprehension and prediction of quantum mechanical phenomena in terms of quasi-distribution functions. During these years, a Monte Carlo technique for the solution of this kinetic equation has been developed, based on the generation and annihilation of signed particles. This technique can be deeply understood in terms of the theory of pure jump processes with a general state space, producing a class of stochastic algorithms. One of these algorithms has been validated successfully by numerical experiments on a benchmark test case.
\end{abstract}

Keywords: Nanostructures, Wigner transport equation, Direct simulation Monte Carlo

AMS subject classification: 82D80, 82S30, 65M75

The Wigner equation is a full quantum transport model able to capture the relevant physics in next generation semiconductor devices. It is well known that the pure state Wigner equation is an equivalent phase-space reformulation of the Schrödinger equation. At the same time the Wigner equation can be augmented by a Boltzmann-like collision operator accounting for the process of decoherence. However, this equation has represented a numerically daunting task and it has raised more problems than solutions. A numerical treatment of the Wigner equation can be dealt with deterministic schemes [1-4], Direct Simulation Monte Carlo [5-8], and it is often used to derive reduced transport models, such as quantum-hydrodynamic models [9-12].

Among the several particle Monte Carlo methods developed during these years (see [13] for a review), we have focused in the so called Signed Monte Carlo method [14], where the Wigner potential is treated as a scat- 


\section{O. Muscato}

tering source which determines the electron-potential interaction, and consequently new particles with different signs are stochastically added to the system. Recently this method has been also be understood in terms of the Markov jump process theory $[15,16]$, producing a class of stochastic algorithms. In this paper we present a thorough validation of one of these algorithms by comparing the Wigner MC results with the Schrödinger equation solution, in an already traditional benchmark experiment. An excellent quantitative agreement is demonstrated despite the very different numerical aspects of the two approaches.

\section{The Wigner function formalism}

In a quantum mechanical set-up the motion of an electron in a pure state is described by the Schrödinger equation

$$
i \hbar \frac{\partial \psi}{\partial t}=H_{x} \psi \quad, \quad H_{x}=-\frac{\hbar^{2}}{2 m^{*}} \Delta_{x}-e V(x, t)
$$

where $x \in \mathbb{R}^{d}$ is the position in the $d$-dimensional Euclidean space, $V(x, t)$ is the external potential, $e$ the absolute value of the electron charge, $m^{*}$ the electron effective mass, $\psi(x, t)$ the normalized wave function, whose square modulus represents the probability density for the position of the electron, i.e.

$$
n_{q}=|\psi(x, t)|^{2} \quad .
$$

The Schrödinger equation is valid for a closed system, but a device is an open system where the contacts and the interactions with the environment (phonons, impurities, etc.) influence the electron state in a way that cannot be described by this equation. The most natural approach to model the statistics in a quantum system is by means of the density function $\rho$, defined as

$$
\rho(r, s, t)=\bar{\psi}(r, t) \psi(s, t) \quad r, s \in \mathbb{R}^{d}
$$

where the diagonal elements represent the probability density $n_{q}$, and the off-diagonal terms characterize the delocalization of electrons (coherence). Differentiating (2) with respect to $t$ and using the Schrödinger equation (1), one obtains the density matrix evolution equation (called Liouville - von Neumann equation)

$$
i \hbar \frac{\partial \rho}{\partial t}=\left(H_{r}-H_{s}\right) \rho
$$

This quantum equation of motion can be reformulated into a kinetic form. If we change the two independent space-variables $(r, s)$ into the center of 


\section{A benchmark study of the Signed-particle Monte Carlo algorithm}

mass one $\left(x, x^{\prime}\right)$

$$
x=\frac{r+s}{2} \quad, \quad x^{\prime}=r-s
$$

we get

$$
\rho\left(x, x^{\prime}, t\right)=\rho\left(x+\frac{x^{\prime}}{2}, x-\frac{x^{\prime}}{2}, t\right) .
$$

Let us suppose that the Fourier transform of $\rho$ with respect to the $x^{\prime}$ variables does exist, then the Wigner function is defined as:

$$
f_{w}(x, k, t)=\frac{1}{(2 \pi)^{d}} \int d x^{\prime} e^{-i k \cdot x^{\prime}} \rho\left(x+\frac{x^{\prime}}{2}, x-\frac{x^{\prime}}{2}, t\right)
$$

where $k \in \mathbb{R}^{d}$ having dimension of the inverse of a length. The fact that the Wigner function depends on the same phase-space variables $(x, k)$, as the Boltzmann distribution function, is remarkable. If we integrate the Wigner function with respect to $k$ we obtain the mean density

$$
n_{q}(x, t)=\int f_{w}(x, k) d k=|\psi(x, t)|^{2} \geq 0
$$

and moreover the first-order moment of the Wigner function, is proportional to the quantum current density of the electron ensemble [17]. Thus, as far as the zeroth and first-order moments are concerned, the Wigner function behaves as the classical particle distribution. However, the Wigner function does not necessarily stay non negative in its evolution process. Unlike in the classical case, it can therefore not be interpreted as a probability density, and it is often referred to as quasi-distribution of particles.

The evolution equation for the Wigner function is found by taking the time derivative of the defining equation (4) and substituting the Liouvillevon Neumann equation (3) on the right-hand side, i.e.

(6) $\frac{\partial f_{w}}{\partial t}+\frac{\hbar}{m^{*}} k \cdot \nabla_{x} f_{w}=\mathcal{Q}\left(f_{w}\right) \quad, \quad \mathcal{Q}\left(f_{w}\right)=\int V_{w}\left(x, k-k^{\prime}\right) f_{w}\left(x, k^{\prime}\right) d k^{\prime}$

where $V_{w}$ is the Wigner potential

$$
V_{w}(x, k)=\frac{1}{i \hbar(2 \pi)^{d}} \int d x^{\prime} e^{-i k \cdot x^{\prime}}\left[V\left(x+\frac{x^{\prime}}{2}\right)-V\left(x-\frac{x^{\prime}}{2}\right)\right] .
$$

The Wigner potential is a non-local potential operator which is responsible of the quantum transport, is real-valued, and anti-symmetric with respect to $k$. So up to now, for a pure state, the Schrödinger equation, the Liouville equation and the Wigner equation are fully equivalent. 


\section{O. Muscato}

But is there any link between the Wigner quantum equation and the classical Boltzmann Transport equation (BTE) ? The answer is yes because, if the potential $V$ is linear, quadratic or varying slowing with respect to typical values of $1 / k$, the quantum evolution term $\mathcal{Q}\left(f_{w}\right)$ reduces to the drift term of the BTE, i.e. [17]

$$
\mathcal{Q}\left(f_{w}\right)=\frac{1}{\hbar} \nabla_{x} V \cdot \nabla_{k} f_{w}(x, k) .
$$

Hence the BTE, without the collisional operator, can be seen as the semiclassical limit of the Wigner equation. Moreover, it is possible to prove that, under some conditions, the collision term describing scattering between electrons and phonons in the BTE, can also be used in the Wigner equation [8] which, in such a case, it is usually called Wigner-Boltzmann transport equation.

\section{The Signed particle Monte Carlo method}

Solving the Wigner equation is a quite difficult task. Much work has been devoted to the direct solution based on finite-difference scheme $[3,4]$. Many difficulties of the direct solution comes from the discretization of the diffusion term $k \cdot \nabla_{x} f_{w}$ because of the typically rapid variation in the phase space. Particle based MC techniques do not require the discretization of this term, and for this reason, they have been developed during these years despite the large computational times $[8,18,19]$.

In the following we shall follow the so called Signed particle Monte Carlo approach developed initially in [14]. This technique is based on the observation that the quantum evolution term $(6)_{2}$ looks like the Gain term of a collisional operator in which the Loss term is missing. But the Wigner potential (7) is not always positive and cannot be considered a scattering term. For this reason, it can be separated into a positive and negative parts $V_{w}^{+}, V_{w}^{-}$such that

$$
V_{w}=V_{w}^{+}-V_{w}^{-} \quad, \quad V_{w}^{+}, V_{w}^{-} \geq 0 \quad .
$$

In this way, we can define an integrated scattering probability per unit time as

$$
\gamma(x)=\int d k^{\prime} V_{w}^{+}\left(x, k-k^{\prime}\right)
$$

and rewrite the quantum evolution term as the difference between Gain and Loss terms, i.e.

$$
\begin{array}{r}
\mathcal{Q}\left(f_{w}\right)=\int d k^{\prime} w\left(k^{\prime}, k\right) f_{w}\left(x, k^{\prime}\right)-\gamma(x) f_{w}(x, k) \\
w\left(k^{\prime}, k\right)=V_{w}^{+}\left(x, k-k^{\prime}\right)-V_{w}^{-}\left(x, k-k^{\prime}\right)+\gamma(x) \delta\left(k-k^{\prime}\right)
\end{array}
$$




\section{A benchmark study of the Signed-particle Monte Carlo algorithm}

Now we can interpret the new scattering rate $w\left(k^{\prime}, k\right)$ and the negative sign in it. For each electron we introduce the weight $A$ called affinity which can be real, and it can be changed according to the scattering rate $w\left(k^{\prime}, k\right)$. In particular:

1. the electron changes its momentum with probability $\simeq V_{w}^{+}$, and its affinity into $+A$

2. the electron changes its momentum with probability $\simeq V_{w}^{-}$, and its affinity into $-A$

3 . the electron does not change momentum with probability $\gamma$, and its affinity does not change. In other words, the initial trajectory is maintained.

Hence the "scattering" mechanism dictated by the $\gamma$ function is interpreted as a new pair particle creation, where the probability that an electron does not produce the new pair (being a markovian process) in $[0, \Delta t[$ is

$$
\begin{aligned}
& \mathbb{P}(\Delta t)=\mathbb{P}(0) \exp \left\{-\int_{0}^{\Delta t} \gamma\left[x\left(t^{\prime}\right)\right] d t^{\prime}\right\}= \\
& \left.\mathbb{P}(0) \exp \left\{-\gamma\left[x\left(t^{\star}\right)\right] \Delta t\right\} \quad, \quad t^{\star} \in\right] 0, \Delta t[
\end{aligned}
$$

likewise to what happens in the BTE [20]. Moreover, if

$$
\gamma\left[x\left(t^{\star}\right)\right] \Delta t<1 \quad \rightarrow \quad \mathbb{P}(\Delta t) \simeq 1-\gamma\left[x\left(t^{\star}\right)\right] \Delta t .
$$

Let be $r$ a uniform random number, if

$$
r<1-\gamma\left[x\left(t^{\star}\right)\right] \Delta t
$$

nothing happens otherwise a couple of new particles is created where the new state is generated from $V_{w}^{+}(x, k)$. In the following we shall use a splitting scheme in order to separate the transport (i.e. movement in the position space) and the creation process, and from eq.(13) $)_{1}$, the splitting time step $\Delta t$ must fulfill

$$
\sup _{x} \gamma[x] \Delta t<1 \quad .
$$

We can summarize the time evolution in the following steps: 


\section{O. Muscato}

\section{1. transport step}

$$
x_{j} \rightarrow x_{j}+\Delta t \frac{\hbar}{m^{*}} k_{j} \quad, \quad j=1, . ., N
$$

The components $A_{j}$ and $k_{j}$ do not change.

\section{2. creation step}

According to probabilistic rules, all particles create new particles that are added to the system.

\section{3. cancellation step}

If the total number of particles exceed a certain threshold $N_{\text {canc }}$, i.e.

$$
N>N_{\text {canc }}
$$

then pairs of particles with similar positions and wave-vectors, but with opposite affinity signs, are removed from the system.

The main drawbacks of this procedure are due to i) efficiency issue in particle generation, because $\gamma$ usually is a rapidly oscillating function, ii) the affinity can take integer values, consequently it yields to an exponential grow of particle numbers, and in step 3) a procedure must be introduced to control the particle number.

\section{Markov jump process theory}

The creation process can be better understood in terms of the Markov jump process theory $[15,16]$. We consider a particle system

$$
z_{j}(t)=\left(A_{j}(t), x_{j}(t), k_{j}(t)\right), \quad t \geq 0 \quad, \quad j=1, . ., N(t)
$$

with the state space $\mathcal{Z}=\cup_{N=1}^{\infty} \mathbb{Z}^{N}$. The single particle state space is

$$
\mathbb{Z}=\mathbb{U} \times \mathbb{R}^{d} \times \mathbb{R}^{d}
$$

where the first component represents a weight $A_{j} \in \mathbb{U}=\{-1,+1\}$, the second component is a position vector, and the third component is a wavevector. Let

$$
\bar{z}=\left(z_{1}, z_{2}, \ldots, z_{N}\right) \in \mathcal{Z} \quad z=(u, x, k) \in \mathbb{Z} .
$$

The time evolution of the system is a pure Markov jump process. Independently of each other, the particles create new particles that are added to the system. The creation rate and the offspring distribution are determined by some kernel $q$. If we introduce the measures

$$
\nu(t, d z)=\mathbb{E}\left(\sum_{j=1}^{N(t)} \delta_{z_{j}(t)}(d z)\right)
$$




\section{A benchmark study of the Signed-particle Monte Carlo algorithm}

it is possible to prove that they satisfy an evolution equation similar to the Dynkin's formula [15], i.e.

(18)

$$
\frac{d}{d t} \int_{\mathbb{Z}} \psi(z) \nu(t, d z)=\int_{\mathbb{Z}} \nu(t, d z) \int_{\mathcal{Z}} q\left(z, d z_{1}, . ., d z_{N}\right)\left[\psi\left(z_{1}\right)+\ldots+\psi\left(z_{N}\right)\right]
$$

where the test functions $\psi(z)$ are continuous with compact support. In our case we have

$$
z=(A, x, k) \quad, \quad \psi(z)=A \varphi(x, k) \quad, \quad f(t, d x, d k)=\int_{\mathbb{U}} A \nu(t, d A, d x, d k)
$$

and then

$$
\int_{\mathbb{Z}} \psi(z) \nu(t, d z)=\int_{\mathbb{R}^{d}} \int_{\mathbb{R}^{d}} \varphi(x, k) f(t, d x, d k)=\mathbb{E}\left(\sum_{j=1}^{N(t)} A_{j}(t) \varphi\left[x_{j}(t), k_{j}(t)\right]\right) .
$$

If $q$ is such that

$$
\begin{array}{r}
\int_{\mathbb{Z}} \nu(t, d z) \int_{\mathcal{Z}} q\left(z, d z_{1}, . ., d z_{N}\right)\left[\psi\left(z_{1}\right)+\ldots+\psi\left(z_{N}\right)\right]= \\
\int_{\mathbb{R}^{d}} \int_{\mathbb{R}^{d}} f(t, d x, d k) \int_{\mathbb{R}^{d}} V_{w}^{(c)}\left(x, k^{\prime}-k\right) \varphi\left(x, k^{\prime}\right) d k^{\prime}
\end{array}
$$

where

$$
V_{w}^{(c)}(x, k)=\left\{\begin{array}{c}
V_{w}(x, k), \quad \text { if }\|k\| \leq c \\
0, \quad \text { otherwise }
\end{array}\right.
$$

and $c>0$ is a cutoff parameter, then eq.(18) writes

$\frac{d}{d t} \int_{\mathbb{R}^{d}} \int_{\mathbb{R}^{d}} \varphi(x, k) f(t, d x, d k)=\int_{\mathbb{R}^{d}} \int_{\mathbb{R}^{d}} f(t, d x, d k) \int_{\mathbb{R}^{d}} V_{w}^{(c)}\left(x, k^{\prime}-k\right) \varphi\left(x, k^{\prime}\right) d k^{\prime}$

which is a weak form of the Wigner equation (6). Functionals of the solution of the Wigner equation (6) are expressed in terms of the particle system (15) using the representation given in (19). This approach has certain advantages compared to other derivations. In particular, it suggests a variety of algorithms and treats these different cases simultaneously. In particular we can consider the following creation kernel:

$$
\begin{array}{r}
\hat{q}(z, d \xi)=\frac{1}{2} \int_{\mathbb{B}_{c}} d k^{\prime} \hat{V}_{w}\left(x, k^{\prime}\right) \times \\
(22)\left[\frac{\left|V_{w}\left(x, k^{\prime}\right)\right|}{\hat{V}_{w}\left(x, k^{\prime}\right)} \delta_{\left(\tilde{A}, x, k+k^{\prime}\right),\left(-\tilde{A}, x, k-k^{\prime}\right)}(d \xi)+\left(1-\frac{\left|V_{w}\left(x, k^{\prime}\right)\right|}{\hat{V}_{w}\left(x, k^{\prime}\right)}\right) \delta_{\emptyset}(d \xi)\right]
\end{array}
$$




\section{O. Muscato}

where $\mathbb{B}_{c}=\left\{k \in \mathbb{R}^{d}:\|k\| \leq c\right\}, \emptyset$ denotes the empty set, the function $\hat{V}_{w}(x, k)$ satisfies

$$
\left|V_{w}(x, k)\right| \leq \hat{V}_{w}(x, k) \quad \forall x, k, \in \mathbb{R}^{d}
$$

and $\tilde{A}$ is defined as

$$
\tilde{A}(A, x, k)=A \operatorname{sign} V_{w}(x, k)
$$

This kernel satisfy the condition (20) and produces the following creation algorithm:

\section{Creation Algorithm $V_{w}$}

1. Let be

$$
\hat{\gamma}(x, c)=\frac{1}{2} \int_{\mathbb{B}_{c}} \hat{V}_{w}(x, k) d k
$$

2. for $j=1, \ldots, N$, with probability

$$
1-\hat{\gamma}\left(x_{j}, c\right) \Delta t
$$

do not create anything. Next particle goto 2

3. otherwise generate randomly a new $\tilde{k}$ according to the probability

$$
\frac{1}{2 \hat{\gamma}\left(x_{j}, c\right)} \hat{V}_{w}\left(x_{j}, k\right) \quad k \in \mathbb{B}_{c}
$$

4. with probability

$$
1-\frac{\left|V_{w}\left(x_{j}, \tilde{k}\right)\right|}{\hat{V}_{w}\left(x_{j}, \tilde{k}\right)}
$$

do not create anything. Next particle goto 2

5 . otherwise create the particle couple

$$
\left[\tilde{A}\left(A_{j}, x_{j}, \tilde{k}_{j}\right), x_{j}, k_{j}+\tilde{k}\right] \quad, \quad\left[-\tilde{A}\left(A_{j}, x_{j}, \tilde{k}_{j}\right), x_{j}, k_{j}-\tilde{k}\right]
$$

with $\tilde{A}$ given in (24). Next particle goto 2 .

\section{The gaussian barrier benchmark}

We have considered a potential barrier with the shape of a gaussian function, centered at $x=0$ with dispersion $\sigma=1 \mathrm{~nm}$ and height $a=0.3 \mathrm{eV}$

$$
V(x)=a \exp \left[-\frac{x^{2}}{2 \sigma^{2}}\right] .
$$




\section{A benchmark study of the Signed-particle Monte Carlo algorithm}

The Wigner potential (7), in the $1 \mathrm{D}$ case, writes

$$
V_{w}(x, k)=\frac{2 a \sigma \sqrt{2 \pi}}{\pi \hbar} \exp \left[-2(\sigma k)^{2}\right] \sin (2 k x) .
$$

The creation algorithm $V_{w}$ is based on the following majorant of $V_{w}(x, k)$

$$
\hat{V}_{w}(x, k)=\frac{2 a \sigma \sqrt{2 \pi}}{\pi \hbar} \quad, \quad \hat{\gamma}(x, c)=\frac{2 a \sigma \sqrt{2 \pi}}{\pi \hbar} c
$$

and the splitting time step condition (14) now yields

$$
\Delta t<\frac{\pi \hbar}{2 a \sigma \sqrt{2 \pi} c} .
$$

The initial condition for the Wigner equation is

$$
f_{w}(x, k, 0)=\frac{1}{\pi} \exp \left[-\frac{\left(x-x_{0}\right)^{2}}{2 \sigma_{0}^{2}}\right] \exp \left[-2 \sigma_{0}^{2}\left(k-k_{0}\right)^{2}\right]
$$

where $x_{0}=-15 \mathrm{~nm}, k_{0}=0.7 \mathrm{~nm}^{-1}, \sigma_{0}=2.852 \mathrm{~nm}$. In the $x$-space we have considered an uniform mesh [-30,30](nm) with $N_{x}=200$ grid-points; also in the $k$-space we have an uniform mesh $[-10,10]\left(\mathrm{nm}^{-1}\right)$ with $N_{k}=400$. We have chosen absorption boundary conditions, i.e. if a particle is out of the mesh then it is erased. The cutoff has been fixed $c=8 \mathrm{~nm}^{-1}$, the initial particle number is $N_{i n i}=160000$, the cancellation parameter $N_{\text {canc }}=$ 480000 , and the time step $\Delta t=0.05$ fsec.

Since in our case the Wigner and Schrödinger equations are equivalent, in order to have a comparison, the Schrödinger equation (1) has been solved using a splitting scheme [21] with initial condition

$$
\psi(0, x)=\frac{1}{\left(2 \pi \sigma_{0}^{2}\right)^{\frac{1}{4}}} \exp \left[-\frac{\left(x-x_{0}\right)^{2}}{4 \sigma_{0}^{2}}\right] \exp \left(i k_{0} x\right) .
$$

The mean density (5), obtained with the two solvers, is shown in figures (1)-(3), for various simulation times.

The agreement between the two solutions is very good. In the figure 4 we plot the particle number in the MC Wigner versus the simulation time: the peaks in this figure are in correspondence with the cancellation procedure. 


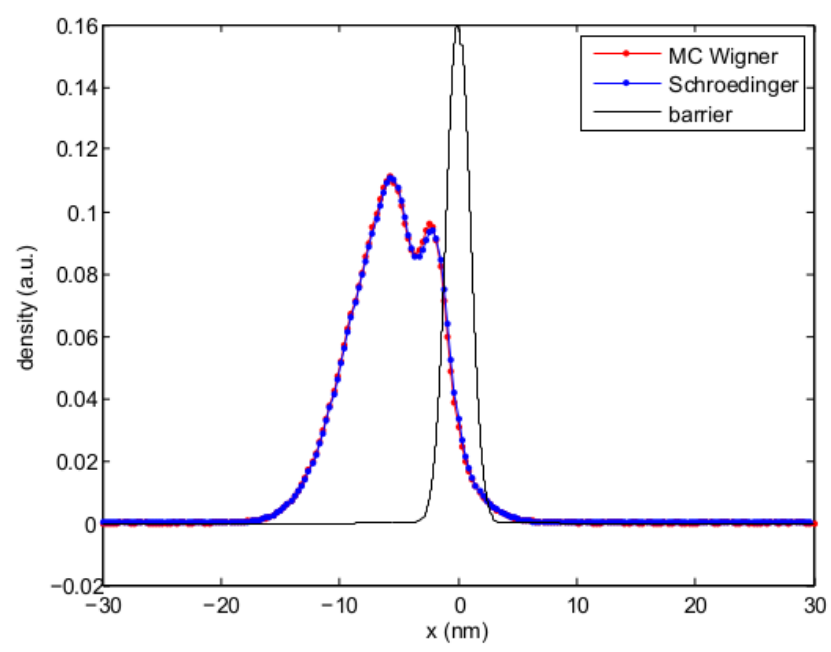

Figure 1. Mean density (5) versus position for $t=8$ fsec.

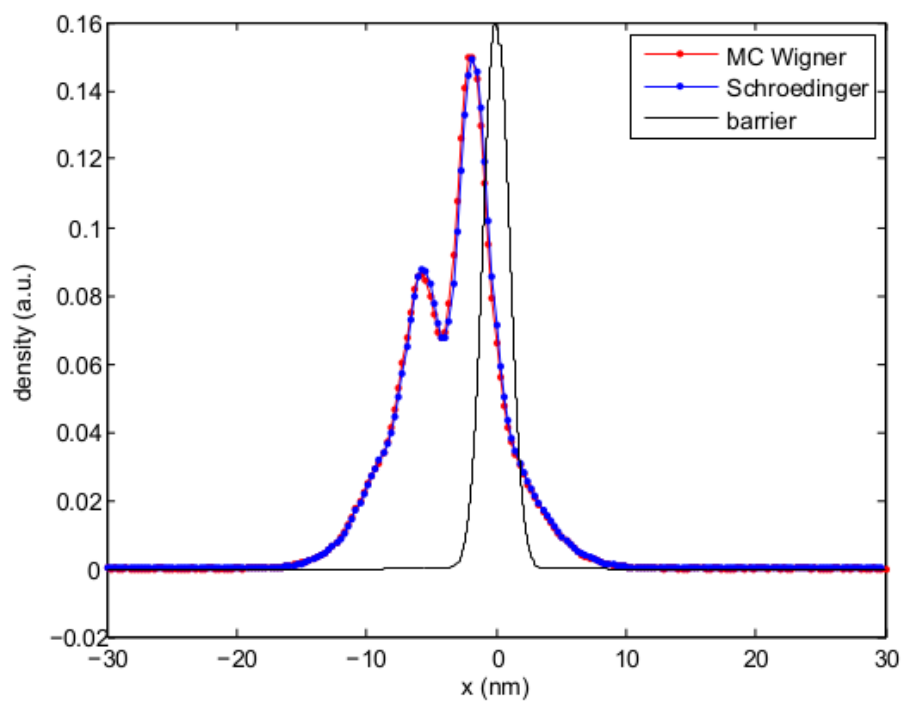

Figure 2. Mean density (5) versus position for $t=10$ fsec.

\section{Conclusions}

The Wigner equation can been solved by using the Signed particle Monte Carlo method, where new pair of particles characterized by a sign are created randomly and added to the system. This creation mechanism 


\section{A benchmark study of the Signed-particle Monte Carlo algorithm}

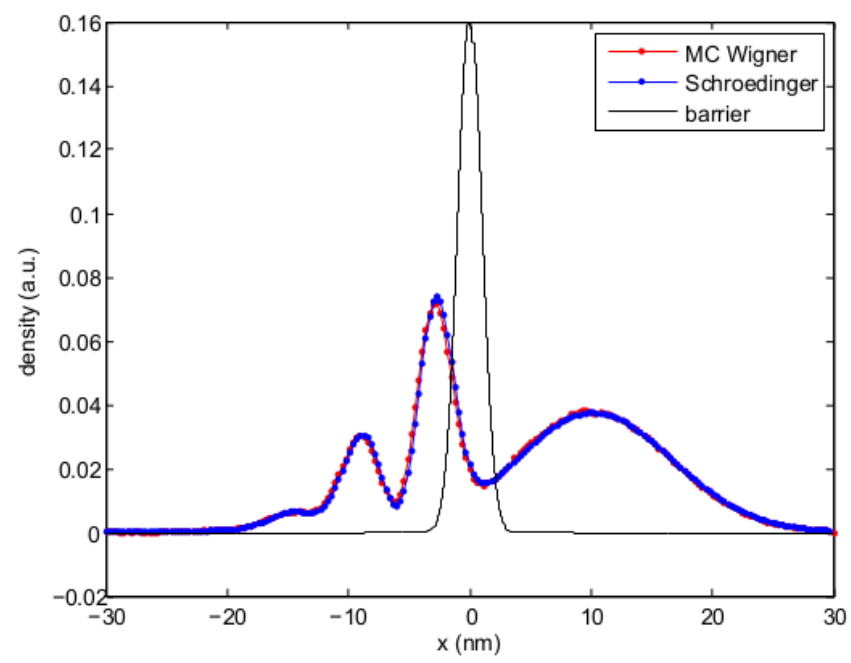

Figure 3. Mean density (5) versus position for $t=20$ fsec.

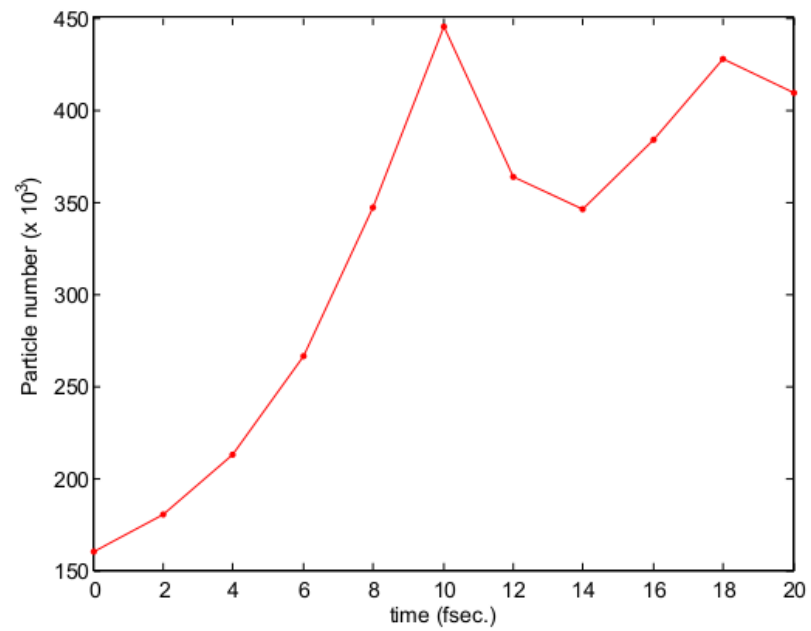

Figure 4. Particle number versus simulation time in the MC Wigner.

has been recently understood in terms of the Markov jump process, producing a class of new stochastic algorithms [16]. One of these algorithms is applied to the gaussian potential barrier benchmark. The results are compared with those obtained by a deterministic splitting scheme for the Schrödinger equation, showing an excellent agreement.

This represents a step ahead toward the simulation of quantum struc- 


\section{O. Muscato}

tures such as silicon nanowire devices [22-25], where the effects of phonon scattering and heating could be included [26-33]. These topics will be the tasks of future researches.

\section{Acknowledgment}

This work has been supported by the National Group of Mathematical Physics (GNFM-INDAM).

\section{REFERENCES}

1. O. Morandi and L. Demeio, A Wigner-function approach to interband transitions based on the multiband-envelope-function model, Transp. Theor. Stat. Phys., vol. 37, no. 5-7, pp. 473-459, 2008.

2. O. Morandi and F. Schürrer, Wigner model for quantum transport in graphene, J. Phys. A: Math. Theor., vol. 26, p. 265301, 2011.

3. S. Shao, T. Lu, and W. Cai, Adaptive conservative cell average spectral element methods for transient Wigner equation in quantum transport, Comm. Comput. Phys., vol. 9, no. 3, pp. 711-739, 2011.

4. A. Dorda and F. Schürrer, A WENO-solver combined with adaptive momentum discretization for the Wigner transport equation and its application to resonant tunneling diodes, J. Comp. Electr., vol. 284, pp. 95-116, 2015.

5. O. Muscato and V. Di Stefano, Heat generation and transport in nanoscale semiconductor devices via Monte Carlo and hydrodynamic simulations, COMPEL, vol. 30, no. 2, pp. 519-537, 2011.

6. O. Muscato, W. Wagner, and V. Di Stefano, Properties of the steady state distribution of electrons in semiconductors, Kinetic and Related Models, vol. 4, no. 3, pp. 809-829, 2011.

7. O. Muscato, V. Di Stefano, and W. Wagner, A variance-reduced electrothermal Monte Carlo method for semiconductor device simulation, Comput. Math. with Appl., vol. 65, no. 3, pp. 520-527, 2013.

8. D. Querlioz and P. Dollfus, The Wigner Monte Carlo method for nanoelectronic devices. Wiley, 2010.

9. P. Degond and C. Ringhofer, Quantum moment hydrodynamics and entropy principle, J. Stat. Phys., vol. 112, no. 3, pp. 587-628, 2003.

10. V. Romano, Quantum corrections to the semiclassical hydrodynamical model of semiconductors based on the maximum entropy principle, $J$. Math. Phys., vol. 48, no. 12, p. 123504, 2007.

11. A. Jüngel, J. Lopez, and J. Montejo Gamez, A new derivation of the quantum Navier-Stokes equations in the Wigner-Fokker-Planck approach, J. Stat. Phys., vol. 145, no. 6, pp. 1661-1673, 2011. 


\section{A benchmark study of the Signed-particle Monte Carlo algorithm}

12. L. Barletti, G. Frosali, and O. Morandi, Kinetic and hydrodynamic models for multi-band quantum transport in crystals, Lect. Notes Comp. Sci. Engineer., vol. 94, pp. 3-56, 2014.

13. H. Kosina, Wigner function approach to nano device simulation, Int. J. Comp. Scien. Engineer., vol. 2, no. 3-4, pp. 100-118, 2006.

14. M. Nedjalkov, H. Kosina, S. Selberherr, C. Ringhofer, and D. K. Ferry, Unified particle approach to Wigner-Boltzmann transport in small semiconductor devices, Phys. Rev. B, vol. 70, p. 115319, 2004.

15. W. Wagner, A random cloud model for the Wigner equation, Kinet. Rel. Models, vol. 9, no. 1, pp. 217-235, 2016.

16. O. Muscato and W. Wagner, A class of stochastic algorithms for the Wigner equation, SIAM J. Sci. Comput., vol. 38, no. 3, pp. A1438A1507, 2016.

17. P. Markovich, C. Ringofer, and C. Schmeiser, Semiconductor equations. Springer-Verlag, 1990.

18. P. Ellinghaus, J. Weinbub, M. Nedjalkov, S. Selberherr, and I. Dimov, Distributed-memory parallelization of the Wigner Monte Carlo method using spatial domain decomposition, Phys. Rev. B, vol. 70, p. 115319, 2004.

19. S. Shao and J. Sellier, Comparison of deterministic and stochastic methods for time-dependent Wigner simulations, J. Comp. Phys., vol. 300, pp. 167-185, 2015.

20. C. Jacoboni and L. Reggiani, The monte carlo method for the solution of charge transport in semiconductors with applications to covalent materials, Rev. Mod. Phys., vol. 55, no. 3, pp. 645-705, 1983.

21. S. Chin and C. Chen, Fourth order gradient symplectic integrator methods for solving the time-dependent schrödinger equation, J. Chem. Phys., vol. 114, pp. 7338-7341, 2001.

22. O. Muscato and V. Di Stefano, Hydrodynamic modeling of silicon quantum wires, J. Comput. Electron., vol. 11, no. 1, pp. 45-55, 2012.

23. O. Muscato and V. Di Stefano, Hydrodynamic simulation of a $n+-n-$ n+ silicon nanowire, Contin. Mech. Thermodyn., vol. 26, pp. 197-205, 2014.

24. O. Muscato and T. Castiglione, Electron transport in silicon nanowires having different cross-sections, Comm. Appl. Ind. Math., vol. 7, no. 2, pp. 8-25, 2016.

25. O. Muscato and T. Castiglione, A hydrodynamic model for silicon nanowires based on the maximum entropy principle, Entropy, vol. 18, no. 10, p. 368, 2016.

26. O. Muscato and V. Di Stefano, Hydrodynamic modeling of the electrothermal transport in silicon semiconductors, J. Phys. A: Math. Theor., 
vol. 44, no. 10, p. 105501, 2011.

27. O. Muscato and V. Di Stefano, An energy transport model describing heat generation and conduction in silicon semiconductors, J. Stat. Phys., vol. 144, no. 1, pp. 171-197, 2011.

28. V. Di Stefano and O. Muscato, Seebeck effect in silicon semiconductors, Acta Appl. Math., vol. 122, no. 1, pp. 225-238, 2012.

29. O. Muscato and V. Di Stefano, Electro-thermal behaviour of a submicron silicon diode, Semicond. Sci. Tech., vol. 28, no. 2, p. 025021, 2013.

30. O. Muscato and V. D. Stefano, Electrothermal transport in silicon carbide semiconductors via a hydrodynamic model, SIAM J. APPL. MATH., vol. 75, no. 4, pp. 1941-1964, 2015.

31. G. Mascali, A hydrodynamical model for silicon semiconductors including crystal heating, Europ. J. Appl. Math., vol. 26, pp. 477-496, 2015.

32. G. Mascali, A new formula for silicon thermal conductivity based on a hierarchy of hydrodynamical models, J. Stat. Phys., vol. 163, no. 5, pp. 1268-1284, 2016.

33. M. Coco, G. Mascali, and V. Romano, Monte Carlo analysis of the thermal effects in monolayer graphene, J. Comp. Theor. Transp., vol. 45, no. 7 , pp. 540-553, 2016. 